\title{
The Evaluation of Exogenous Melatonin Administration in Supraspinatus Overuse Tendinopathy in an Experimental Rat Model
}

\author{
Onur Kocadal ${ }^{\varpi}$, Murad Pepe ${ }^{1}$, Nalan Akyurek² ${ }^{2}$ Zafer Gunes ${ }^{3}$, Hatice Surer ${ }^{4}$, Ertugrul Aksahin ${ }^{5}$, Betul Ogut ${ }^{6}$, \\ Cem Nuri Aktekin ${ }^{7}$
}

Department of Orthopedics and Traumatology, Yeditepe University Faculty of Medicine, Istanbul, ${ }^{1}$ Department of Orthopedics and Traumatology, Alanya Research and Training Hospital, Alanya Alaaddin Keykubat Unversity, Alanya, ${ }^{2}$ Department of Pathology, Gazi University Medical School, Ankara, ${ }^{3}$ Department of Orthopedics and Traumatology, Bursa Yüksek Ihtisas Research and Training Hospital, Bursa, ${ }^{4}$ Department of Biochemistry and Clinical Biochemistry, Ankara Education and Research Hospital, ${ }^{5}$ Department of Orthopedics and Traumatology, Medical Park Hospital, Ankara, ${ }^{6}$ Department of Pathology, Gaziantep Cengiz Gokcek Obstetrics and Children's Hospital, Gaziantep, ${ }^{7}$ Department of Orthopedics and Traumatology, Ankara Education and Research Hospital, Ankara, Turkey

Background: Increased oxidative stress and inflammation play a critical role in the etiopathogenesis of chronic tendinopathy. Melatonin is an endogenous molecule that exhibits antioxidant and anti-inflammatory activity. The aim of this study was to evaluate the biochemical and histopathological effects of exogenous melatonin administrations in supraspinatus overuse tendinopathy.

Methods: Fifty rats were divided into the following four groups: cage activity, melatonin treatment, corticosteriod therapy, and control. Melatonin $(10 \mathrm{mg} / \mathrm{kg}$, intraperitoneal; twice a day) and triamcinolone $(0.3 \mathrm{mg} / \mathrm{kg}$, subacromial; weekly) were administered to the treatment groups after the overuse period. Biochemical and histopathological evaluations were performed on serum samples and biopsies obtained from rats. Plasma inducible nitric oxide synthase (iNOS), vascular endothelial growth factor (VEGF), total antioxidant status (TAS), total oxidant status (TOS), and oxidative stress index (OSI) levels were evaluated biochemically.

Results: The TAS, TOS, OSI, iNOS, and VEGF values were significantly lower than the pre-treatment levels in rats receiving exogenous melatonin treatment ( 3 or 6 weeks) $(p<0.05)$. TOS, iNOS, VEGF, and OSI values after 3 weeks of triamcinolone administration, and TOS, VEGF, and OSI levels after 6 weeks of triamcinolone application, were significantly lower than the pre-treatment levels $(p<0.05)$. Conclusions: Exogenous melatonin application in overuse tendinopathy reduces oxidative stress and inflammation. Melatonin might be an alternative potential molecule to corticosteroids in the treatment of chronic tendinopathy.

(Clin Shoulder Elbow 2019;22(2):79-86)

Key Words: Shoulder; Rotator cuff; Tendinopathy; Oxidative stress; Melatonin

\section{Introduction}

Shoulder overuse tendinopathy is a pathological disorder that disrupts the quality of life and is commonly diagnosed in athletes and people with recurrent overhead activities. ${ }^{1)}$ Overuse tendinopathy is most commonly observed in the supraspinatus tendon. ${ }^{2,3)}$ Chronic tendinopathy causes some alterations such as degeneration of collagen structure, hyperangiogenesis, increased cellularity and biomechanical weakening of the tendon. ${ }^{3,4)}$ In chronic tendinopathy, free radicals such as nitric oxide $(\mathrm{NO})$ are released in high amounts due to increased oxidative stress. ${ }^{5}$

Chronic tendinopathy treatments aim to reduce inflammation and oxidative stress and prevent the development of irreversible degeneration. Conservative treatment methods include corticosteroid injections, nonsteroidal anti-inflammatory drugs, extracorporeal shock wave therapy, platelet-rich plasma, and

Received January 11, 2019. Revised March 7, 2019. Accepted March 12, 2019.

Correspondence to: Onur Kocadal

Department of Orthopedics and Traumatology, Yeditepe University Kozyatağı Hospital, İçerenköy Mahallesi Hastane Yolu Sokak no.102-104, Ataşehir 34752, Istanbul, Turkey

Tel: +90-5323364878, Fax: +90-2164693796, E-mail: onurkocadal@gmail.com, ORCID: https://orcid.org/0000-0002-7390-6888

IRB approval: Ankara Education and Research Hospital (No. 261).

Financial support: This study was supported by grants from the Turkish Orthopedics and Traumatology Association-Turkish Orthopedic Research Council (date: 03.03.2015; number: 04) for their contributions to this project. Conflict of interests: None. 
physiotherapy regimens. ${ }^{6}$ Subacromial corticosteroid injections are an option in the treatment of chronic tendinopathy. However, corticosteroids are reported to trigger complications such as tendon rupture, subcutaneous atrophy, avascular necrosis, dermatitis, skin depigmentation, and periarticular calcifications. ${ }^{7}$

Melatonin is an antioxidant and anti-inflammatory molecule secreted from the pineal gland. ${ }^{8)}$ The protective effects of melatonin have been demonstrated in pathologies such as crush injury or ischemia-reperfusion damage, where increased oxidative stress plays a key role as an etiological factor. ${ }^{910)}$ To the best of our knowledge, there is no study reported in literature evaluating the effects of melatonin in overuse tendinopathy. The aim of this experimental study was to evaluate the histopathological and biochemical effects of melatonin administration in supraspinatus overuse tendinopathy.

\section{Methods}

\section{Study Design}

This study was approved by the Ankara Research and Training Hospital Ethical Committee for Experimental Animals (date: 06.03.2014; No. 261). Fifty-five male Sprague-Dawley rats weighing 350 to $400 \mathrm{~g}$ were used. The rats were housed at room temperature under 12-hour light/dark conditions, and fed ad libitum. Throughout the study period, all rats were examined daily by a veterinarian. In the pilot study, to ensure that subacromial injections were administered at the correct location, diluted methylene blue injections were administered to 5 randomly selected rats. Shoulder dissection performed after injections confirmed that all injections were accurately administered in the subacromial space. ${ }^{11)}$
The remaining 50 rats constituted the study groups; 8 rats were left in cage activity (CA) as a sham group for evaluation of overuse activity. For the development of overuse tendinopathy in the remaining 42 rats, the adaptation exercise consisted of treadmill walking at a speed of $17 \mathrm{~m} / \mathrm{min}, 10$ minutes a day for 2 weeks, and 5 days a week. Subsequently, the rats were exposed to a downhill run on a $10^{\circ}$ decline running platform (Bama Healthcare, Ankara, Turkey) for 4 weeks, 5 days a week, at a speed of $17 \mathrm{~m} / \mathrm{min}^{\left.3{ }^{3}\right)}$ These 42 rats with overuse activity were divided randomly into 3 groups of 14 rats each, and assigned to melatonin treatment $(\mathrm{M})$, corticosteroid therapy (CS), and control (C) groups. Following the overuse activity period, rats were left in normal cage activities. Pharmacological therapy was administered to rats for 3 weeks (M-3, CS-3) or 6 weeks (M-6, CS-6) after completion of the overuse activity. Melatonin (Sigma Chemical Corp., St Louis, MO, USA) was dissolved in $1 \%$ ethanol and diluted with $0.09 \mathrm{NaCl}$ solution. It was administered intraperitoneally at a dose of $10 \mathrm{mg} / \mathrm{kg}$ every 12 hours. ${ }^{12)}$ Triamcinolone (Kenacort-A; Deva Holding, Istanbul, Turkey) was diluted using $0.09 \mathrm{NaCl}$ solution for corticosteroid injection; $0.3 \mathrm{mg} / \mathrm{kg}$ triamcinolone was injected once a week to the left subacromial area. ${ }^{11)}$ No other pharmacological treatment was administered to the other control rats (CA, C-3, C-6). After 3 weeks, 7 rats from each group were randomly selected (M-3, CS-3, C-3) for biochemical and histopathological evaluation; the animals were euthanized under general anesthesia after biopsy. Biochemically and histopathologic examination of the remaining 7 rats from each group (M-6, CS-6, C-6) was similarly completed at 6 weeks after the overuse activity (Fig. 1).

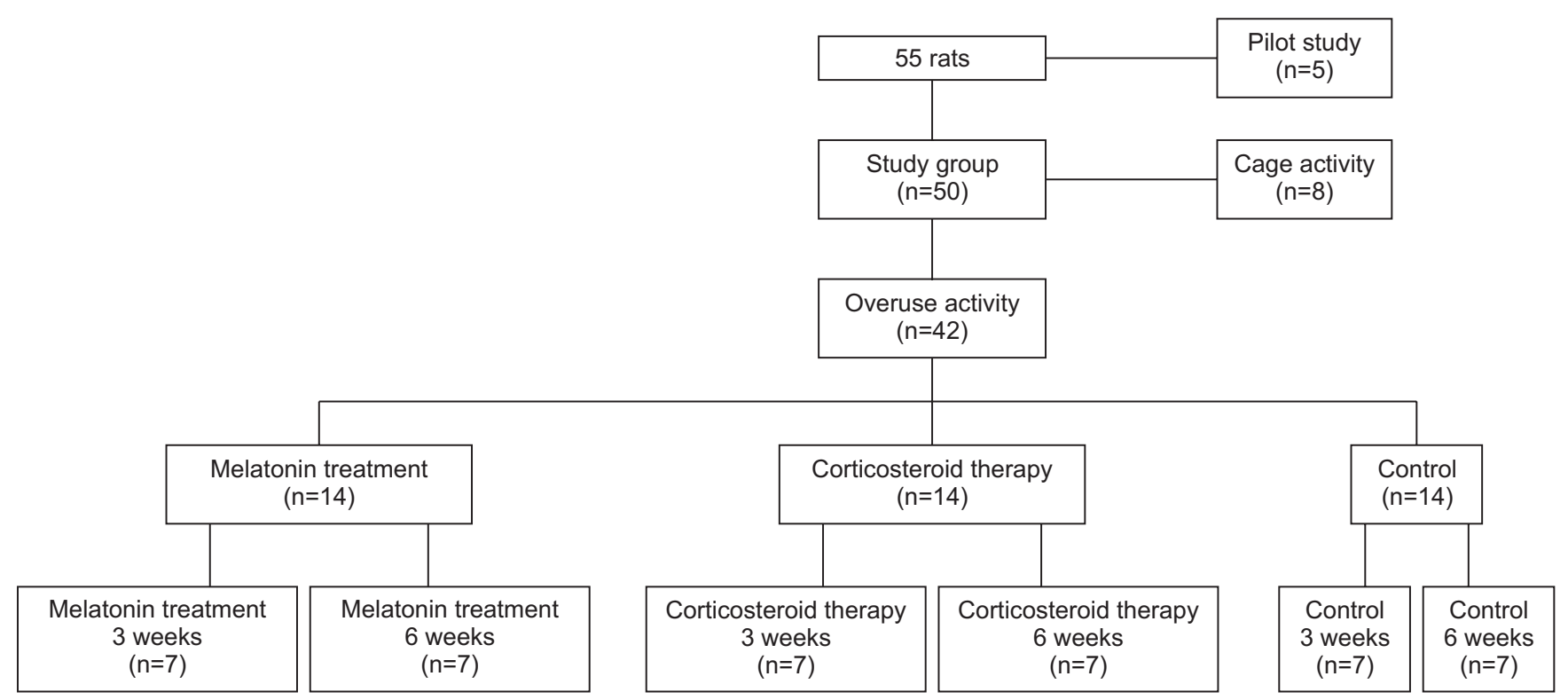

Fig. 1. Flowchart of the study design. 


\section{Biochemical Evaluations}

For biochemical evaluation, blood samples were collected from all experimental rats $(n=50)$ following termination of the overuse model. Blood samples were centrifuged at 1,000 $\times g$ for 15 minutes at $4{ }^{\circ} \mathrm{C}$ in the laboratory, and the obtained serum samples were stored at $-80^{\circ} \mathrm{C}$ until further analysis of their vascular endothelial growth factor (VEGF), total antioxidant status (TAS), total oxidant status (TOS), oxidative stress index (OSI), and inducible nitric oxide synthase (iNOS) levels.

\section{Evaluation of Total Antioxidant Status}

Plasma TAS levels were measured using the commercial kit developed by Erel (TAS Assay Kit; Rel Assay Diagnostics, Gaziantep, Turkey). ${ }^{13)}$ This method is based on the principle of reducing the dark blue-green colored 2,2'-azinobis-3-ethylbenzothiazoline-6-sulfonic acid antioxidant radical in the obtained samples to the colorless form. The absorbance change of samples was measured spectrophotometrically at $660 \mathrm{~nm}$ wavelength, and expressed as mmol Trolox equivalent/L. ${ }^{14)}$

\section{Evaluation of Total Oxidant Status}

Plasma TOS levels were measured using a commercial kit (TOS Assay Kit; Rel Assay Diagnostics). This technique is based on the principle that oxidants in the obtained samples oxidize the ferrous ion-chelator complex to the ferric ions, resulting in the coloration of formed ferric ions with chromogenic material in an acidic medium. ${ }^{15)}$ The absorbance change of samples was measured spectrophotometrically at $530 \mathrm{~nm}$ wavelength, and expressed as $\mu$ mol $\mathrm{H}_{2} \mathrm{O}_{2}$ equivalent/L.

The levels of TAS and TOS were measured using an auto analyzer (Olympus AU680; Beckman Coulter, Miami, FL, USA). The ratio of the TOS level to the TAS level was calculated as OSI, using the formula: OSI (arbitrary unit) $=\left[\mathrm{TOS}\left(\mathrm{mmol} \mathrm{H}_{2} \mathrm{O}_{2}\right.\right.$

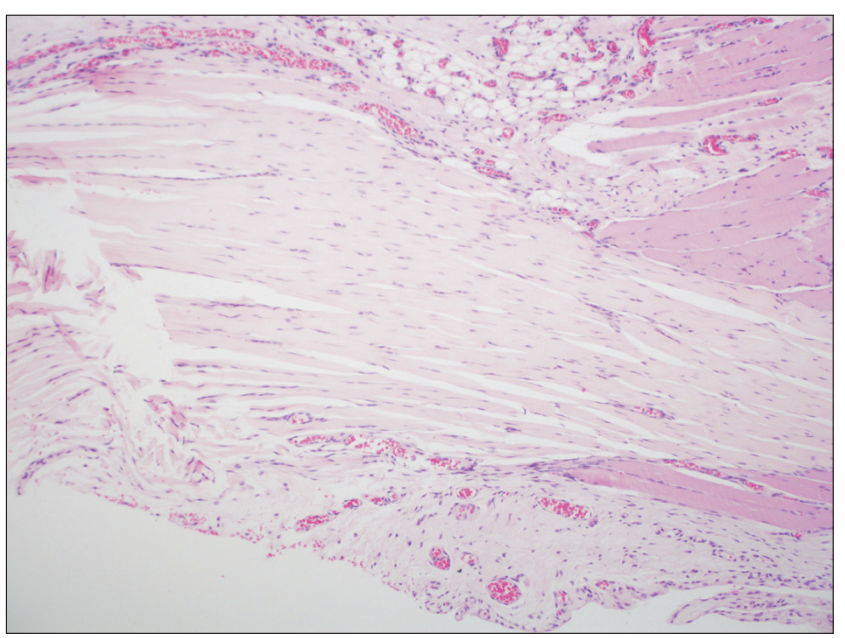

Fig. 2. Histopathologic section with normal cellularity and collagen orientation (grade 0$)(\mathrm{H} \& \mathrm{E}, \times 100)$.
equivalent/L)/TAS (mmol Trolox equivalent/L)/100]. ${ }^{16)}$

\section{Evaluation of Inducible Nitric Oxide Synthase}

The iNOS levels were measured by the sandwich enzymelinked immunosorbent assay (ELISA). Plasma iNOS levels were analyzed with commercially available assay kits (Rat iNOS Nitric Oxide Synthase Inducible ELISA Kit; Elabscience Biotechnology Corp., Ltd., Wuhan, China). The results were expressed in ng/ $\mathrm{ml}$.

\section{Evaluation of Vascular Endothelial Growth Factor}

VEGF levels were measured by sandwich ELISA. Plasma VEGF levels were analyzed with commercially available assay kits (Rat Vascular Endothelial cell Growth Factor ELISA Kit; Elabscience Biotechnology Corp., Ltd.). The results were expressed in $\mathrm{ng} / \mathrm{ml}$. Plasma VEGF and iNOS levels were measured using an ELISA microplate strip washer (ELX50; BioTek Instruments, Winooski, VT, USA) and an ELISA microplate reader (ELX800; BioTek Instruments).

\section{Histopathological Evaluations}

For histopathological evaluation of the overuse activity, the right supraspinatus tendon of all experimental rats was biopsied (50 rats) on completion of the overuse activity. In the CA group (8 rats), rats were euthanized under general anesthesia after biopsy. Briefly, rats were anesthetized with xylazine hydrochloride (7 mg/ $/ \mathrm{kg}$ ) and ketamine hydrochloride $(60 \mathrm{mg} / \mathrm{kg}$ ). The biopsy procedure was then performed under sterile conditions. Once anesthetized, an incision was made over the spine of scapula. The extensions of trapezius and deltoid in this region were released. After biopsy, the deltoid and trapezius muscles were sutured back to the scapular spine. ${ }^{17)}$ The second biopsy was performed using the left supraspinatus tendon to assess the treat-

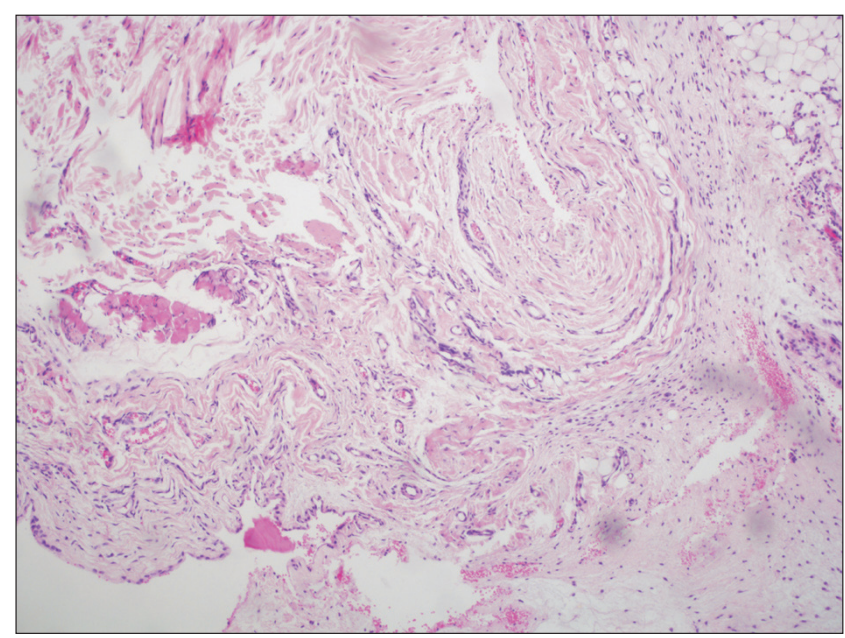

Fig. 3. Histopathologic section presenting severe deterioration of collagen orientation, increased cellularity and deformation of cell shape (grade 3) $(\mathrm{H} \& \mathrm{E}, \times 100)$. 
ment efficacy at 3 or 6 weeks following the first biopsy of the rats undergoing overuse activity. The biopsy samples were fixed in $10 \%$ buffered formalin solution, following which the specimens were embedded in paraffin. All specimens were stained with hematoxylin and eosin and analyzed under a light microscope by a pathologist who was blinded to the study groups. Histopathological evaluation of the supraspinatus tendon was performed as per the staging system described by Soslowsky et al. ${ }^{17)}$ Changes in the supraspinatus tendon were assessed by considering 'cellularity, fibroblastic changes, collagen fiber organization and disruption'. Scoring in the staging system is considered as follows: 0 , normal; 1 , mild change; 2 , moderate change; and 3, marked change (Fig. 2, 3).

\section{Statistical Analysis}

Statistical analysis was performed using IBM SPSS ver. 21 (IBM Corp., Armonk, NY, USA) for Mac. The Wilcoxon signed-rank test was used to perform intra-group comparisons. The MannWhitney U-test was applied to compare scale data between two groups. Chi-square test and generalized McNemar's test compared the histopathological parameters. A $p$-value of less than 0.05 is accepted as statistically significant.

\section{Results}

Results of the biochemical and histopathological evaluations of all groups are summarized in Table 1, 2 .

\section{Evaluation of Overuse Activity}

The mean TOS and VEGF values in rats with overuse activity were statistically higher than the CA group $(p<0.05)$. The histopathological stages were significantly different between the group of rats exposed to overuse activity and CA group $(p=0.04)$ (Table 1).

\section{Comparison within Each Group}

The mean values of TAS, TOS, OSI, iNOS, and VEGF after treatment in the M-3 and M-6 groups were statistically and significantly lower than the pre-treatment period $(p<0.05)$.

The mean TOS, iNOS, VEGF, and OSI values after treatment in the CS-3 group were statistically and significantly lower than the pre-treatment period $(p<0.05)$. In the CS- 6 group, the mean TOS, VEGF, and OSI levels after treatment were significantly lower as compared to the pre-treatment period $(p<0.05)$.

The TOS and OSI values 3 weeks after overuse activity (group C-3) and the TAS and VEGF values after 6 weeks of overuse activity (group C-6) were statistically and significantly lower $(p<0.05)$ (Table 2).

\section{Discussion}

This study demonstrates that melatonin reduces oxidative damage by decreasing the OSI and iNOS levels in the experimental rat overuse tendinopathy model. In addition, it further demonstrates that melatonin treatment results in a decrease in VEGF levels. These results indicate that melatonin may be an alternative molecule to subacromial corticosteroid injections in the treatment of overuse tendinopathy.

The etiopathogenesis of the commonly encountered chronic tendinopathies is still not fully understood. Recurrent trauma, anatomic features such as acromial shape, age-related tendon damage, acute and chronic inflammation, vascular disorders, and oxidative stress induced tenocyte apoptosis are some of the factors implicated in the development of tendinopathy. ${ }^{18)}$ Overuse activity is also a frequently associated factor involved in the development of chronic tendinopathy. ${ }^{2,18}$ In recent years, there has been increased interest in experimental studies for evaluating the etiopathogenesis and treatment modalities of supraspinatus tendinopathy associated with overuse. We used the rat overuse model in this study, since the shoulder anatomy of rats is very similar to that of humans. ${ }^{11,17)}$

Table 1. Evaluation of Overuse Activity

\begin{tabular}{lcccc}
\hline \multicolumn{1}{c}{ Variable } & Overuse activity $(\mathrm{n}=42)$ & Cage activity $(\mathrm{n}=8)$ & All rats $(\mathrm{n}=50)$ & $p$-value \\
\hline iNOS $(\mathrm{ng} / \mathrm{ml})$ & $0.69 \pm 0.16(0.5-1.24)$ & $0.75 \pm 0.05(0.69-0.85)$ & $0.70 \pm 0.15(0.5-1.24)$ \\
VEGF $(\mathrm{ng} / \mathrm{ml})$ & $179.3 \pm 222.3(32-1,054)$ & $76.7 \pm 69.5(32-208.0)$ & $162.2 \pm 207.9(32-1,054)$ & 0.10 \\
TAS $(\mathrm{mmol} \mathrm{Trolox} \mathrm{equivalent/L)}$ & $1.54 \pm 0.18(1.24-1.99)$ & $1.40 \pm 0.17(1.18-1.74)$ & $1.5 \pm 0.18(1.18-1.99)$ \\
TOS $\left(\mu \mathrm{mol} \mathrm{H}_{2} \mathrm{O}_{2}\right.$ equivalent/L) & $22.8 \pm 11.6(7.5-61.4)$ & $14.6 \pm 7.2(4.8-28.7)$ & $21.4 \pm 11.3(4.8-61.4)$ \\
OSI (arbitrary unit) & $1,456.6 \pm 646.8(514.0-3,430.7)$ & $1,015.3 \pm 389.1(339.5-1,654.6)$ & $1,381.5 \pm 629.8(339.5-3,430.7)$ & 0.05 \\
Histopathological stage & $1.05 \pm 0.8(0-3)$ & $0.75 \pm 1.1(0-3)$ & $1 \pm 0.8(0-3)$ & $0.03^{*}$ \\
\hline
\end{tabular}

Values are presented as mean \pm standard deviation (range). Mann-Whitney U-test was performed for comparison of biochemical parameters between the study groups. The chi-square test was used to compare histopathological parameters between the groups.

iNOS: inducible nitric oxide synthase, VEGF: vascular endothelial growth factor, TAS: total antioxidant status, TOS: total oxidant status, OSI: oxidative stress index.

${ }^{*}$ Statistically significant $(p<0.05)$. 
Table 2. Evaluation of Pre- and Post-treatment Biochemical and Histopathological Parameters of All Rats in Overuse Activity

\begin{tabular}{|c|c|c|c|c|c|c|}
\hline Group & iNOS (ng/ml) & VEGF (ng/ml) & $\begin{array}{c}\text { TAS (mmol Trolox } \\
\text { equivalent/L) }\end{array}$ & $\begin{array}{c}\mathrm{TOS}\left(\mu \mathrm{mol} \mathrm{H}_{2} \mathrm{O}_{2}\right. \\
\text { equivalent } / \mathrm{L})\end{array}$ & $\begin{array}{c}\text { OSI } \\
\text { (arbitrary unit) }\end{array}$ & $\begin{array}{c}\text { Histopathological } \\
\text { stage (G0:G1:G2:G3) }\end{array}$ \\
\hline \multicolumn{7}{|l|}{ M-3 wk } \\
\hline Pre-treatment & $0.65(0.58-0.78)$ & $116(43-236)$ & $1.58(1.3-1.8)$ & $18.7(13.3-31.9)$ & $1,203.7(948.2-1,814.5)$ & $3: 3: 0: 1$ \\
\hline Post-treatment & $0.52(0.49-0.58)$ & $38(32-57)$ & $1.16(0.8-1.3)$ & $4.2(2.1 \pm 7.5)$ & $364.6(261.7-669.0)$ & $5: 0: 2: 0$ \\
\hline$p$-value & $0.02^{*}$ & $0.01^{*}$ & $0.01^{*}$ & $0.01^{*}$ & $0.01^{*}$ & 0.4 \\
\hline \multicolumn{7}{|l|}{ M-6 wk } \\
\hline Pre-treatment & $0.82(0.56-1.13)$ & $115(32-416)$ & $1.58(1.24-1.75)$ & $15.5(11.8 \pm 35.6)$ & $1,001(957.2-2,037.7)$ & $1: 3: 0: 3$ \\
\hline Post-treatment & 0.56 & 32.5 & 1.34 & 4.9 & 417.8 & $2: 5: 0: 0$ \\
\hline$p$-value & $0.04^{*}$ & 0.04 & $0.04^{*}$ & $0.02^{*}$ & $0.02^{*}$ & 0.05 \\
\hline \multicolumn{7}{|l|}{ CS-3 wk } \\
\hline Pre-treatment & $0.75(0.55-1.24)$ & $136(66-825)$ & $1.45(1.29-1.99)$ & $20.3(10.2-61.4)$ & $1,430.5(514.0-3,430.7)$ & $1: 6: 0: 0$ \\
\hline Post-treatment & $0.56(0.50-0.69)$ & $37(32-54)$ & $1.35(1.32-1.53)$ & $5.8(4.5-7.4)$ & $429(333.0-509.5)$ & $4: 2: 1: 0$ \\
\hline$p$-value & $0.04^{*}$ & $0.02^{*}$ & 0.2 & $0.02^{*}$ & $0.02^{*}$ & 0.3 \\
\hline \multicolumn{7}{|l|}{ CS-6 wk } \\
\hline Pre-treatment & $0.59(0.53-0.84)$ & $180(40-1054)$ & $1.55(1.26-1.75)$ & $20(11.8-45.1)$ & $1,471.7(943.6-2,704.7)$ & 1:5:1:0 \\
\hline Post-treatment & $0.55(0.53-0.72)$ & $37(32-55)$ & $1.4(1.12-1.48)$ & $5.6(2.9-15.3)$ & $381.6(235.7-1,007.8)$ & $1: 5: 1: 0$ \\
\hline$p$-value & 0.3 & $0.01^{*}$ & 0.1 & $0.01^{*}$ & $0.01^{*}$ & $<0.999$ \\
\hline \multicolumn{7}{|l|}{ C-3 wk } \\
\hline Pre-treatment & $0.59(0.53-0.81)$ & $79(32-204)$ & $1.52(1.32-1.74)$ & $24.3(19.3-41.5)$ & $1,601.5(1,463.6-2,413.9)$ & $3: 4: 0: 0$ \\
\hline Post-treatment & $0.54(0.51-0.62)$ & $34(32-58)$ & $1.32(1.22-1.5)$ & $5.4(4.0 \pm 6.5)$ & $400(313.6-515.5)$ & $4: 3: 0: 0$ \\
\hline$p$-value & 0.1 & 0.06 & 0.08 & $0.04^{*}$ & $0.02^{*}$ & 0.3 \\
\hline \multicolumn{7}{|l|}{ C-6 wk } \\
\hline Pre-treatment & $0.58(0.50-0.79)$ & $58(38-156)$ & $1.5(1.32-1.89)$ & $13.2(7.5-25.4)$ & $893.9(569.7-1,477.9)$ & $0: 6: 0: 1$ \\
\hline Post-treatment & $0.56(0.53-0.58)$ & $33(32-36)$ & $1.22(0.97-1.45)$ & $4.6(2.9-16.7)$ & $339(301.0-1,481.4)$ & $4: 1: 1: 1$ \\
\hline$p$-value & 0.2 & $0.01^{*}$ & $0.02^{*}$ & 0.06 & 0.12 & 0.1 \\
\hline
\end{tabular}

Values are presented as median (range) or mean \pm standard deviation. Wilcoxon signed-rank test was used for the comparison of biochemical parameters in each group and generalized McNemar's test was used to compare histopathological parameters.

iNOS: inducible nitric oxide synthase, VEGF: vascular endothelial growth factor, TAS: total antioxidant status, TOS: total oxidant status, OSI: oxidative stress index, M: melatonin treatment group, CS: corticosteroid therapy group, C: control group.

${ }^{*}$ Statistically significant $(p<0.05)$.

Experimental studies have reported various histopathologic changes in the supraspinatus tendon after overuse activity, such as collagen structure irregularity, increased cell count and vascular structure irregularities." Similar to other researches in literature, this study confirms the development of histopathological disorders in rats after overuse activity. Increase in the metabolic activity due to exercise leads to increased oxidative stress, ${ }^{16)}$ which in turn is implicated in literature as one of the factors involved in the development of tendinopathy. ${ }^{19,20)}$ This study demonstrates that the oxidative load is increased in rats with chronic overuse activity.

Melatonin, an endogenous indolamine, has regenerative and protective properties in peripheral tissues. ${ }^{9}$ The protective effect of melatonin on peripheral tissues is indicated by inhibi- tion of the production of free oxygen radicals, the activation of antioxidant enzymes and a synergistic effect with other antioxidants. ${ }^{10,211}$ Experimental studies have demonstrated the antiinflammatory and antioxidant effects of melatonin in conditions such as crush injury, acute exercise, and ischemia-reperfusion injury, where increased oxygen radicals and widespread inflammation play a role in the physiopathology. ${ }^{10,22)}$ In this study, the role of melatonin in the treatment of chronic tendinopathy was explained by considering the role of inflammation and increased oxidative stress in the pathogenesis of overuse tendinopathy.

$\mathrm{NO}$, a diatomic, highly reactive free radical, is produced by the nitric oxide synthases (NOS) enzyme family. ${ }^{5}$ ) The NOS enzyme family comprises of the iNOS, endothelial NOS, and neuronal NOS forms. ${ }^{8}$ The effects of NO vary according to concen- 
tration levels. High levels of NO are associated with increased inflammatory response, increased oxidative stress, cytotoxicity and metalloproteinase activation. ${ }^{5,23)}$ Szomor et al. ${ }^{5)}$ reported an increase in iNOS mRNA expression in samples obtained from the supraspinatus muscles of rats after 4 weeks of overuse activity. In the current study, no significant difference was found in plasma iNOS levels in rats subjected to overuse activity. This may be due to the fact that systemic iNOS levels do not reflect the expression of iNOS in the local tissue. One important effect of melatonin is the inhibition of $\mathrm{NO}$ production. ${ }^{8)}$ Experimental studies have reported that melatonin reduces the anti-inflammatory and antioxidant effects by decreasing iNOS expression in peripheral tissues. ${ }^{24)}$ Our study shows that the application of exogenous melatonin in rat overuse tendinopathy reduces the plasma iNOS levels. To the best of our knowledge, this study is the first to evaluate application of exogenous melatonin in a rat overuse model. It has also been reported in literature that corticosteroids inhibit iNOS expression in peripheral tissues. ${ }^{25}$ In the current study, serum iNOS levels were decreased in the CS-3 group. However, in contrast to the CS-3 group, iNOS levels in the CS-6 group did not change when compared to the pre-treatment levels. This condition may be related to the timedependent effects of corticosteroids, and the change in the response of the damaged tissue to corticosteroids as the treatment process progresses. Thus, it can be said that melatonin and subacromial corticosteroid injections have a similar effect on iNOS regulation.

Histopathologically, degeneration and repair are seen concurrently in overused tendons. Some parts of the tendon reveal microtears in the collagen bundle, hypocellularity, fibrocartilaginous metaplasia and necrosis, while hypercellularity, fibrosis, and fibroblastic proliferation are seen in other areas. ${ }^{5}$ One of the pathological changes in overuse tendinopathy is hypervascularity. VEGF, a potent angiogenic cytokine, is responsible for the increased vascularity in overuse tendinopathy. ${ }^{4)}$ Perry et al. ${ }^{1)}$ reported that overuse tendinopathy in rats resulted in increased VEGF expression. Increased VEGF expression has also been reported in patients with patellar tendinopathy. ${ }^{4)}$ In our study, the plasma VEGF levels in rats subjected to overuse activity were assessed to be about 2-fold higher than the CA group.

Corticosteroids are frequently used in the treatment of chronic inflammatory diseases. One of the most important effects of corticosteroids is to inhibit the expression of multiple inflammatory genes. ${ }^{26)}$ Suppression of VEGF, which plays a critical role in the etiopathogenesis of chronic inflammatory diseases, is one of the appropriate therapeutic approaches. ${ }^{27)}$ Thus, corticosteroids are a suitable pharmacological agent for the treatment of chronic tendinopathy. VEGF is a component of the physiological inflammatory response in the early period. ${ }^{4)}$ Therefore, similar to poldeoxyribonucleotides, VEGF plays an important role in triggering angiogenesis in the early phase of tissue injury healing. ${ }^{28,29)}$
Therefore, these factors need to be considered in corticosteroid treatment.

Recent studies concerned with tumoral angiogenesis have discussed the association of melatonin with VEGF. ${ }^{30)}$ It has been reported that melatonin suppresses VEGF expression in cancerous tissues. ${ }^{31)}$ Our study similarly shows that exposure to melatonin reduces the plasma VEGF levels in the rat overuse tendinopathy model. A similar relationship in this study was also observed in rats receiving triamcinolone therapy. In literature, experimental studies have reported that corticosteroids inhibit the VEGF induced angiogenesis. ${ }^{32,33)}$ Reducing VEGF release using melatonin and corticosteroids may therefore be critical for controlling hyperangiogenesis, which is one of the components of tendinopathy.

The most appropriate factor indicating the relationship between oxidant and antioxidant status is OSI. ${ }^{16,34)}$ Antioxidant status is directly related to oxidative stress. This study suggests that OSI levels decrease significantly in rats receiving melatonin treatment. Moreover, antioxidant status was also unexpectedly decreased in these rats, which may possibly be due to decreased oxidative stress.

In this study, overuse activity resulted in significant histopathological changes in the tendon structure. Melatonin and corticosteroid therapy exert histopathological effects on the structure of the tendon, which are positive but not statistically significant. We suggest a few reasons for this situation. Damage to the tendon after overuse may be reversible or irreversible. Other shoulder evaluation for biopsy may have caused the histopathological evaluation to be suboptimal. One of possible reason of minimal histological improvement may be that the grade of tendinopathy in experimental group is not severe. Hence, the treatment effect may not be up to expectation. In this study, tendinopathy could be severe by designing a study using models in which the impingement syndrome is developed by combining intrinsic and extrinsic mechanisms. ${ }^{17,35)}$

Melatonin has a plasma half-life of about half an hour, which makes it highly safe and non-toxic even at high doses. ${ }^{36)}$ For this reason, in our study, we assessed the application of exogenous melatonin as 3 or 6 weeks apart. Plasma OSI, iNOS, and VEGF levels were decreased in both the 3- and 6-week administrations of melatonin. On the other hand, there was no general difference between the 3- and 6-week applications of melatonin. No toxic effects were observed in any rat during the 6-week pharmacological treatment. Therefore, we believe that melatonin may be suitable for long time treatment of chronic tendinopathy in rats.

This study has some limitations. Although the anatomy of the shoulder of rats is similar to that of humans, the development of tendinopathy may not be identical due to the characteristics of walking on four legs. Second limitation is that the histopathological evaluation of the rats was made from the contralateral ten- 
don. This method was chosen to avoid histopathologic misevaluation due to possible fibrosis, adhesions and inflammation developed in tissues after the initial surgery. Another limitation of the study is that the hematological parameters in the C-3 and C- 6 groups do not overlap. In the C-3 and C- 6 groups, all hematological parameters were decreased after surgery compared to the preoperative period. However, some results were not statistically significant. This condition may be related to the relatively small sample size for subgroup analysis. The objective biochemical and histopathologic evaluation of the overuse model, allowing for short and long term applications, can be considered the strong points of the study.

\section{Conclusion}

Exogenous melatonin administration leads to a decrease in plasma iNOS, VEGF, and oxidative stress parameters. Melatonin may be a potent molecule in the treatment of chronic tendinopathy with its antioxidant and anti-inflammatory activity.

\section{References}

1. Perry SM, Mcllhenny SE, Hoffman MC, Soslowsky LJ. Inflammatory and angiogenic mRNA levels are altered in a supraspinatus tendon overuse animal model. J Shoulder Elbow Surg. 2005;14(1 Suppl S):79-83S. doi: 10.1016/j.jse.2004.09.020.

2. Attia M, Huet E, Gossard C, Menashi S, Tassoni MC, Martelly I. Early events of overused supraspinatus tendons involve matrix metalloproteinases and EMMPRIN/CD147 in the absence of inflammation. Am J Sports Med. 2013;41(4):908-17. doi: 10.1177/0363546512473817.

3. Soslowsky LJ, Thomopoulos S, Tun S, et al. Neer Award 1999. Overuse activity injures the supraspinatus tendon in an animal model: a histologic and biomechanical study. J Shoulder Elbow Surg. 2000;9(2):79-84.

4. Scott A, Lian $\varnothing$, Bahr R, Hart DA, Duronio V. VEGF expression in patellar tendinopathy: a preliminary study. Clin Orthop Relat Res. 2008;466(7):1598-604. doi: 10.1007/s11999-0080272-x.

5. Szomor ZL, Appleyard RC, Murrell GA. Overexpression of nitric oxide synthases in tendon overuse. J Orthop Res. 2006; 24(1):80-6. doi: 10.1002/jor.20009.

6. Loppini M, Maffulli N. Conservative management of tendinopathy: an evidence-based approach. Muscles Ligaments Tendons J. 2012;1(4):134-7.

7. Nichols AW. Complications associated with the use of corticosteroids in the treatment of athletic injuries. Clin J Sport Med. 2005;15(5):370-5.

8. Mauriz JL, Collado PS, Veneroso C, Reiter RJ, González-Gallego J. A review of the molecular aspects of melatonin's antiinflammatory actions: recent insights and new perspectives.
J Pineal Res. 2013;54(1):1-14. doi: 10.1111/j.1600-079X. 2012.01014.x.

9. Stratos I, Richter N, Rotter R, et al. Melatonin restores muscle regeneration and enhances muscle function after crush injury in rats. J Pineal Res. 2012;52(1):62-70. doi: 10.1111/j.1600079X.2011.00919.x.

10. Erkanli K, Kayalar N, Erkanli G, Ercan F, Sener G, Kirali K. Melatonin protects against ischemia/reperfusion injury in skeletal muscle. J Pineal Res. 2005;39(3):238-42. doi: 10.1111/ j.1600-079X.2005.00240.x.

11. Tillander B, Franzén LE, Karlsson MH, Norlin R. Effect of steroid injections on the rotator cuff: an experimental study in rats. J Shoulder Elbow Surg. 1999;8(3):271-4. doi: 10.1016/ S1058-2746(99)90141-6.

12. Hong $\mathrm{Y}$, Kim $\mathrm{H}$, Lee $\mathrm{Y}$, et al. Salutary effects of melatonin combined with treadmill exercise on cartilage damage. J Pineal Res. 2014;57(1):53-66. doi: 10.1111/jpi.12143.

13. Erel O. A novel automated direct measurement method for total antioxidant capacity using a new generation, more stable ABTS radical cation. Clin Biochem. 2004;37(4):277-85. doi: 10.1016/j.clinbiochem.2003.11.015.

14. Atmaca N, Atmaca HT, Kanici A, Anteplioglu T. Protective effect of resveratrol on sodium fluoride-induced oxidative stress, hepatotoxicity and neurotoxicity in rats. Food Chem Toxicol. 2014;70:191-7. doi: 10.1016/j.fct.2014.05.011.

15. Erel O. A new automated colorimetric method for measuring total oxidant status. Clin Biochem. 2005;38(12):1103-11. doi: 10.1016/j.clinbiochem.2005.08.008.

16. Kurkcu R, Cakmak A, Zeyrek D, Atas A, Karacabey K, Yamaner F. Evaluation of oxidative status in short-term exercises of adolescent athletes. Biol Sport. 2010;27(3):177-80.

17. Soslowsky LJ, Carpenter JE, DeBano CM, Banerji I, Moalli MR. Development and use of an animal model for investigations on rotator cuff disease. J Shoulder Elbow Surg. 1996;5(5):38392. doi: 10.1016/S1058-2746(96)80070-X.

18. Nho SJ, Yadav H, Shindle MK, Macgillivray JD. Rotator cuff degeneration: etiology and pathogenesis. Am J Sports Med. 2008;36(5):987-93. doi: 10.1177/0363546508317344.

19. Bestwick CS, Maffulli N. Reactive oxygen species and tendon problems: review and hypothesis. Sports Med Arthrosc Review. 2000;8(1):6-16.

20. Yuan J, Murrell GA, Trickett A, Wang MX. Involvement of cytochrome $\mathrm{c}$ release and caspase- 3 activation in the oxidative stress-induced apoptosis in human tendon fibroblasts. Biochim Biophys Acta. 2003;1641(1):35-41. doi: 10.1016/S01674889(03)00047-8

21. López-Burillo S, Tan DX, Mayo JC, Sainz RM, Manchester LC, Reiter RJ. Melatonin, xanthurenic acid, resveratrol, EGCG, vitamin $\mathrm{C}$ and alpha-lipoic acid differentially reduce oxidative DNA damage induced by Fenton reagents: a study of their individual and synergistic actions. J Pineal Res. 2003;34(4):269- 
77. doi: 10.1034/j.1600-079X.2003.00041.x.

22. Veneroso C, Tuñón MJ, González-Gallego J, Collado PS. Melatonin reduces cardiac inflammatory injury induced by acute exercise. J Pineal Res. 2009;47(2):184-91. doi: 10.1111/ j.1600-079X.2009.00699.x.

23. Cho NS, Hwang JH, Lee YT, Chae SW. Tendinosis-like histologic and molecular changes of the achilles tendon to repetitive stress: a pilot study in rats. Clin Orthop Relat Res. 2011;469(11):3172-80. doi: 10.1007/s11999-011-2000-1.

24. Alonso M, Collado PS, González-Gallego J. Melatonin inhibits the expression of the inducible isoform of nitric oxide synthase and nuclear factor kappa B activation in rat skeletal muscle. J Pineal Res. 2006;41(1):8-14. doi: 10.1111/j.1600-079X.2006. 00323.x.

25. Matsumura M, Kakishita H, Suzuki M, Banba N, Hattori Y. Dexamethasone suppresses iNOS gene expression by inhibiting NF-kappaB in vascular smooth muscle cells. Life Sci. 2001;69(9):1067-77. doi: 10.1016/S0024-3205(01)01196-1.

26. Barnes PJ. Anti-inflammatory actions of glucocorticoids: molecular mechanisms. Clin Sci (Lond). 1998;94(6):557-72. doi: 10.1042/cs0940557.

27. Pufe T, Petersen WJ, Mentlein R, Tillmann BN. The role of vasculature and angiogenesis for the pathogenesis of degenerative tendons disease. Scand J Med Sci Sports. 2005;15(4):211-22. doi: 10.1111/j.1600-0838.2005.00465.x.

28. Kim JK, Chung JY. Effectiveness of polydeoxyribonucleotide injection versus normal saline injection for treatment of chronic plantar fasciitis: a prospective randomised clinical trial. Int Orthop. 2015;39(7):1329-34. doi: 10.1007/s00264-015-27720.

29. Ryu K, Ko D, Lim G, Kim E, Lee SH. Ultrasound-guided prolotherapy with polydeoxyribonucleotide for painful rotator cuff tendinopathy. Pain Res Manag. 2018;2018:8286190. doi:

\subsection{5/2018/8286190.}

30. Lissoni P, Rovelli F, Malugani F, Bucovec R, Conti A, Maestroni G). Anti-angiogenic activity of melatonin in advanced cancer patients. Neuro Endocrinol Lett. 2001;22(1):45-7.

31. Carbajo-Pescador $S$, Ordoñez R, Benet $M$, et al. Inhibition of VEGF expression through blockade of Hif1 $\alpha$ and STAT3 signalling mediates the anti-angiogenic effect of melatonin in HepG2 liver cancer cells. Br J Cancer. 2013;109(1):83-91. doi: 10.1038/bjc.2013.285.

32. Ebrahem Q, Minamoto A, Hoppe G, Anand-Apte B, Sears JE. Triamcinolone acetonide inhibits IL-6- and VEGF-induced angiogenesis downstream of the IL-6 and VEGF receptors. Invest Ophthalmol Vis Sci. 2006;47(11):4935-41. doi: 10.1167/ iovs.05-1651.

33. Greenberger S, Boscolo E, Adini I, Mulliken JB, Bischoff J. Corticosteroid suppression of VEGF-A in infantile hemangiomaderived stem cells. N Engl J Med. 2010;362(11):1005-13. doi: 10.1056/NEJMoa0903036.

34. Ahsen A, Gonul Y, Genc A, et al. Protective effect of melatonin on infrarenal aortic occlusion: this effect is related to antiinflammatory effect and antioxidant effect. Inflammation. 2014;37(4):1111-9. doi: 10.1007/s10753-014-9835-Z.

35. Carpenter JE, Flanagan CL, Thomopoulos S, Yian EH, Soslowsky LJ. The effects of overuse combined with intrinsic or extrinsic alterations in an animal model of rotator cuff tendinosis. Am J Sports Med. 1998;26(6):801-7. doi: 10.1177/03635465980260061101.

36. Borges Lda S, Dermargos A, da Silva Junior EP, Weimann E, Lambertucci RH, Hatanaka E. Melatonin decreases muscular oxidative stress and inflammation induced by strenuous exercise and stimulates growth factor synthesis. J Pineal Res. 2015;58(2):166-72. doi: 10.1111/jpi.12202. 